COMMENT. Fukuyama-type congenital muscular dystrophy is frequently complicated by cardiac involvement after 10 years of age. Treatment with ACE inhibitors and, if needed, beta-blockers, when patients develop early signs of left ventricular dysfunction, may delay the onset of heart failure. Routine cardiac evaluation, including echocardiograms and follow-up, is recommended, especially in older children.

Treatment of the heart in Duchenne muscular dystrophy (DMD) is discussed in an editorial (Baxter P. Dev Med Child Neurol March 2006;48:163-163). Multicenter trials are recommended, since reports of treatment with ACE inhibitors and steroids are variable. Cardiomyopathy develops in most patients with DMD by age 18 years, and is the leading cause of death in $10-40 \%$. Initiating therapy at the pre-symptomatic stage of LV dysfunction may delay the onset of cardiac failure and improve prognosis (Bourke JP. Cardiac monitoring and treatment for children and adolescents with neuromuscular disorders. Dev Med Child Neurol March 2006;48:164-164).

\title{
DIAGNOSIS AND TREATMENT OF DERMATOMYOSITIS
}

The impact of duration of untreated symptoms in children with juvenile dermatomyositis (JDM) on clinical and laboratory findings at diagnosis was studied in 166 patients enrolled in the National JDM Research Registry and examined at Children's Memorial Hospital, Northwestern University; Loyola University; and University of Chicago, Chicago, IL; and other centers in the US. The girl:boy ratio was $2: 1 ; 74 \%$ white, $13 \%$ Hispanic, and 10\% African-American. Duration of untreated disease ranged from .07 month to 98 months (median, 4.04 months). Age at diagnosis and duration of disease showed no significant differences with gender or race. Rash (heliotrope eyelids, malar erythema, Gottron's metacarpal papules) was the first symptom reported by 100 patients (65\%), and muscle weakness was the first in $44(29 \%)$. Nine $(6 \%)$ had both rash and weakness at disease onset. The severity of muscle symptoms based on disease activity scores (DAS 0-11) was greater in nonwhite (6.77) $\mathrm{cf}$ white (4.71) children $(\mathrm{P}>.0005)$. Mean DAS total (skin rash and muscle weakness) was higher in nonwhite (12.52) $\mathrm{cf}$ white $(10.45)$ children $(\mathrm{P}=.001)$. DAS skin rash (scale 0-9) ranged from 3-9 (mean, 6) and did not vary with duration of untreated disease, whereas DAS muscle weakness (scale 0-11) ranged from 0-11 and declined gradually with duration of untreated disease $(\mathrm{P}>.0005)$. More children with untreated JDM were in lower percentiles for height and weight than their healthy controls, irrespective of the duration of untreated disease. In addition to rash and muscle weakness, symptoms at diagnosis of JDM in order of frequency included capillary dilation and telangiectasia, followed by arthritis, dysphagia, and abdominal pain. Dysphagia and arthritis were more common in older children, whereas cutaneous calcifications were correlated with duration of untreated disease $(\mathrm{P}=.006)$. Levels of all 4 routine muscle enzymes $(\mathrm{CK}$, aldolase, $\mathrm{LDH}$, and SGOT/AST) were lower with longer duration of untreated disease; they were normal in 13$26 \%$ of children at the initial clinic visit. CK level was normal in 33 untreated JDM cases, despite muscle weakness. (Pachman LM, Abbott K, Sinacore JM, et al. Duration of illness is an important variable for untreated children with juvenile dermatomyositis. J Pediatr Feb 2006;148:247-253). (Reprints: Dr Lauren M Pachman, Children's Memorial Medical Center, Division of Immunology/Rheumatology, 2300 Children's Plaza, Box 50, Chicago, IL 60614). 
COMMENT. Duration of disease and age are important in reviewing the symptoms and serum enzymes at time of diagnosis of JDM. Children with longer disease duration tend to have fewer diagnostic findings of JDM, and their muscle enzymes are often normal. Older children have more symptoms of arthritis and dysphagia compared to children age 6 years and under. Systemic vasculopathy, with decreased food absorption, and dysphagia may account for the lower percentile height and weight of children with JMD. Early diagnosis and prompt institution of treatment are recommended.

Dysphagia in facioscapulohumeral muscular dystrophy (FSHD) was demonstrated in 7 of 8 adults, most having weakness of tongue and jaw muscles. (Wohlgemuth $\mathrm{M}$ et al. Neurology 2006;66:1926-1928). This finding is contrary to the original description of FSHD by Landouzy and Dejeurine in 1885, emphasizing the integrity of the muscles of the tongue and jaw. Dysphagia appears to occur only in severe cases of FSHD, and does not involve muscles of the pharynx.

\section{SEIZURE DISORDERS}

\section{HYPOGLYCEMIA AND SEIZURES IN LARGE FULL-TERM NEONATES}

The risk of hypoglycemia-associated seizures in large-for-gestational-age (LGA) fullterm neonates was assessed by analysis of data from the Netherlands Perinatal Registry at Wilhelmina Children's Hospital, Utrecht. Hypoglycemia, defined as a plasma glucose below $2.5 \mathrm{mmol} / \mathrm{l}$, was recorded from $1997-2002$ in 1513 of $9318(16.2 \%)$ of LGA full-term neonates without maternal diabetes; $20(1.3 \%)$ of these had seizures, and hypoglycemia was the single cause of seizures in 6 . Perinatal asphyxia, birth trauma and infections may have contributed to the hypoglycemia and seizures in the remaining 14. Hyperinsulinism was demonstrated in one LGA neonate with seizures and maternal diabetes, and in none without diabetes. The number with abnormal neurodevelopment was not recorded. The safe lower level of plasma glucose in healthy LGA neonates needs to be established. (Groenendaal F, Elferink-Stinkens PM, \& the Netherlands Perinatal Registry. Hypoglycaemia and seizures in large-for-gestational-age (LGA) full-term neonates. Acta Paediatrica July 2006;95:874876). (Respond: Floris Groenendaal, Department of Neonatology, Room KE 04.123.1, Wilhelmina Children's Hospital/University Medical Center, Lundlaan 6, 3584 EA Utrecht, the Netherlands).

COMMENT. Hypoglycemic seizures may occur in LGA full-term neonates even in the absence of risk factors, such as maternal diabetes, perinatal asphyxia, trauma or infections, but the incidence is low. A wide variation in definition of hypoglycemia is quoted in textbooks, ranging from a glucose level of $<1$ to $<2.5 \mathrm{mmol} / \mathrm{l}$ for term babies of appropriate weight. An even greater range is used by practicing pediatricians (Koh THHG et al. Neonatal hypoglycemia - the controversy regarding definition Arch Dis Child 1988;63:1386-1398). The safe blood glucose concentration varies with many factors, including rate of fall of blood glucose. (Etheridge JE Jr. Hypoglycemia and the central 